\title{
ON $n$-WEBS OF CURVES IN A PLANE
}

BY G. BOL

This note contains a proof of Theorem 4 of the list given by W. Blaschke* in a preceding paper.

If $t_{i}=$ const. represents $n$ sheaves of curves in a plane, then the maximal number of linearly independent relations

$$
\sum_{i} U_{i k}\left(t_{i}\right)=0, \quad(k=1, \cdots, m, i=1, \cdots, n),
$$

is

$$
N=\frac{1}{2}(n-1)(n-2) .
$$

Let (1) be any set of such relations; then we consider $U_{i k}\left(t_{i}\right)$, $(k=1, \cdots, m)$, for a fixed $i$ to be the $m$ coordinates of a point describing a curve $p_{i}\left(t_{i}\right)$ in an affine $m$-space.

If we can prove that the curves $p_{i}\left(t_{i}\right)$ all lie in parallel linear subspaces of dimension $N$, our theorem is proved, for this means that between the coordinates of every $p_{i}$ there exist linear relations with the same constant coefficients, which express $m-N$ of the coordinates in terms of the other $N$. And this means that of the $m$ relations (1) there can be only $N$ linearly independent.

If we assume our functions $U_{i k}$ to be differentiable a suitable number of times, however, this last statement comes down to proving that among the vectors

$$
\frac{d}{d t_{i}} p_{i}\left(t_{i}\right)=p_{i}{ }^{\prime}\left(t_{i}\right), \quad p_{i}^{\prime \prime}\left(t_{i}\right), \quad p_{i}^{\prime \prime \prime}\left(t_{i}\right), \cdots,
$$

there cannot be more than $N$ linearly independent ones. $\dagger$

We will prove this for $n=5, N=6$; the proof can easily be extended to all values of $n$. To avoid the use of many indices, we will write (1) in the form

$$
p_{1}(u)+p_{2}(v)+p_{3}(r)+p_{4}(s)+p_{5}(t)=0 .
$$

* W. Blaschke, Results and problems about n-webs of curves in a plane, this Bulletin, vol. 38 (1932), p. 828.

$\dagger$ This does not really make it necessary to assume the functions (1) to be analytic; from a certain order $m$ we can always replace (3) by an existence statement for solutions of a differential equation. 
As our parameters $t_{i}$ are given functions of the coordinates in the plane of our curves, and are all independent functions, we can express them as functions of $u$ and $v$. We then differentiate the vector equation (3) with respect to $u$ and $v$ and find

$$
\begin{array}{ll}
0=p_{1}^{\prime} & +p_{3}^{\prime} r_{u}+p_{4}^{\prime} s_{u}+p_{5}^{\prime} t_{u}, \\
0= & p_{2}^{\prime} \quad+p_{3}^{\prime} r_{v}+p_{4}^{\prime} s_{v}+p_{5}^{\prime} t_{v} \\
0=p_{1}^{\prime \prime} & +p_{3}^{\prime \prime} r_{u}^{2}+p_{4}^{\prime \prime} s_{u}^{2}+p_{5}^{\prime \prime} t_{u}^{2}+p_{3}^{\prime} r_{u u}+p_{4}^{\prime} s_{u u}
\end{array}
$$

(8) $0=p_{3}^{\prime \prime} r_{u} r_{v}+p_{4}^{\prime \prime} s_{u} s_{v}+p_{5}^{\prime \prime} t_{u} t_{v}+p_{3}^{\prime} r_{u v}+p_{4}^{\prime} s_{u v}+p_{5}^{\prime} t_{u v}$,

$$
0=p_{3}^{\prime \prime \prime} r_{u}^{2} r_{v}+p_{4}^{\prime \prime \prime} s_{u}{ }^{2} s_{v}+p_{5}^{\prime \prime \prime} t_{u}{ }^{2} t_{v}+L\left(p_{i}^{\prime \prime}, p_{i}^{\prime}\right) \text {; }
$$

(9) $0=p_{3}^{\prime \prime \prime} r_{u} r_{v}^{2}+p_{4}^{\prime \prime \prime} s_{u} s_{v}^{2}+p_{5}^{\prime \prime \prime} t_{u} t_{v}^{2}+L\left(p_{i}^{\prime \prime}, p_{i}^{\prime}\right)$,

$$
0=p_{3}{ }^{\mathrm{iv}} r_{u}{ }^{3} r_{v}+p_{4}{ }^{\mathrm{iv}} s_{u}{ }^{3} s_{v}+p_{5}{ }^{\mathrm{iv}} t_{u}{ }^{3} t_{v}+L\left(p_{i}^{\prime \prime \prime}, p_{i}^{\prime \prime}, p_{i}^{\prime}\right)
$$

(10) $0=p_{3}{ }^{\mathrm{iv}} r_{u}{ }^{2} r_{v}{ }^{2}+p_{4}{ }^{\mathrm{iv}} s_{u}{ }^{2} s_{v}{ }^{2}+p_{5}{ }^{\mathrm{iv}} t_{u}{ }^{2} t_{v}{ }^{2}+L\left(p_{i}^{\prime \prime \prime}, p_{i}^{\prime \prime}, p_{i}^{\prime}\right)$,

$0=p_{3}{ }^{\mathrm{iv}} r_{u} r_{v}{ }^{2}+p_{4}{ }^{\mathrm{iv}} s_{u} s_{v}{ }^{3}+p_{5}{ }^{\mathrm{iv}} t_{u} t_{v}{ }^{3}+L\left(p_{i}^{\prime \prime \prime}, p_{i}^{\prime \prime}, p_{i}^{\prime}\right) ;$

Here $L$ always means a linear combination of the vectors in brackets, and $i=3,4,5$. In this way we get two groups of equations. The first expresses all the derivatives of $p_{1}$ and $p_{2}$ as combinations of those of $p_{3}, p_{4}$, and $p_{5}$. The latter can be used to prove that of these there can be no more than 6 linearly independent.

If we assume for a moment that the equations (8), (9), (10) are not in a disturbing way dependent, then the result is obvious. For then we can have at the utmost 3 independent vectors $p_{i}^{\prime}$, of the vectors $p_{i}^{\prime \prime}$ one can be expressed by means of the others and $p_{i}^{\prime}$ as a consequence of (8), so we get only two extra independent vectors, and (9) shows that vectors $p_{i}^{\prime \prime \prime}$ can give only one extra dimension. The total number is exactly $3+2+1=6$. 
So the only thing that remains to be proved is that relations (8), (9), (10) are really independent. Now in (8) the coefficients of $p_{i}^{\prime \prime}$ cannot vanish. For $r_{u}=0$ would mean that $r$ was a function of $u$ alone, and therefore that sheaves $r=$ const. and $u=$ const. would coincide. So (8) really gives us a relation between the $p_{i}^{\prime \prime}$. To show that (9) gives 2 relations we have to consider the matrix

$$
\left\|\begin{array}{ccc}
r_{u}^{2} r_{v} & s_{u}^{2} s_{v} & t_{u}^{2} t_{v} \\
r_{u} r_{v}^{2} & s_{u} s_{v}^{2} & t_{u} t_{v}^{2}
\end{array}\right\|
$$

and show that it is of rank 2. But one of the determinants is

$$
r_{u} r_{v} s_{u} s_{v} \cdot\left|\begin{array}{cc}
r_{u} & s_{u} \\
r_{v} & s_{v}
\end{array}\right|
$$

and none of the factors can vanish, the last one since this would imply the dependence of the functions $r$ and $s$, which is again impossible. Finally the essential determinant in (10) is equal to

$$
r_{u} r_{v} s_{u} s_{v} t_{u} t_{v}\left|\begin{array}{cc}
r_{u} & s_{u} \\
r_{v} & s_{v}
\end{array}\right|\left|\begin{array}{cc}
s_{u} & t_{u} \\
s_{v} & t_{v}
\end{array}\right|\left|\begin{array}{cc}
r_{u} & t_{u} \\
r_{v} & t_{v}
\end{array}\right|
$$

so that from (10) we can really compute $p_{i}{ }^{i v}$ as linear combinations of $p_{i}^{\prime \prime \prime}, p_{i}^{\prime \prime}, p_{i}^{\prime}$. We see that there is no danger for dependency of the equations, and our theorem is proved.

Of course if $n>5$, we have a similar proof, only the determinants we have to consider are of higher order. We find $N=n-2+n-3+n-4+\cdots+2+1=\frac{1}{2}(n-1)(n-2)$.

As a corollary, for $n=4$, we have: If a 2-dimensional surface in $k$-space can be generated in two different ways as a translation surface, it lies in a linear three-dimensional subspace.*

For the assumption leads to a vector equation (4) with $n=4$ and our formula gives $N=3$.

The University of Chicago, ANd

The University of Hamburg

* See S. Lie, Leipziger Berichte, 1897, p. 186. 